\title{
The Value of Productivity in Health Policy
}

\author{
Wilbert Van den Hout ${ }^{1}$
}

Published online: 28 April 2015

(c) Springer International Publishing Switzerland 2015

In this issue of Applied Health Economics and Health Policy, Pearce and her co-authors estimate productivity losses associated with head and neck cancer [1]. Like previous research, they show that the human capital approach (HCA) and the friction cost approach (FCA) render vastly different estimates of the value of productivity loss. In addition, they show that results differ by the type of productivity loss (such as temporary time off and loss due to mortality) and by socio-demographic and clinical variables (such as age and type of treatment). The paper contributes to the continuing debate about whether and how the value of productivity should be used in health policy.

\section{The human capital and friction cost approaches}

Disease and treatment can have a profound impact on productivity. Apart from personal satisfaction, purpose, and income, productivity also provides welfare to society which may compensate for the costs of healthcare (although savings on productivity are more easily promised than proven). As the value of productive output is too hard to measure, current methods instead measure the value of the productive input: the HCA and FCA both measure worked hours and value those according to the wage rate. With reference to free-market economy, the value of the patients' productive input should be similar to the value of the output.

Wilbert Van den Hout

w.b.van_den_hout@lumc.nl

1 Department of Medical Decision Making and Quality of Care, Leiden University Medical Center, PO Box 9600, 2300 RC Leiden, The Netherlands
The HCA and FCA differ in the worked hours and the additional costs they take into account. The underlying assumption of the HCA is full employment, so each working hour can be put to good use. Thus, every reduction in working hours up to the patient's retirement age can be considered as costs to society. The fundamental idea of the FCA is that adaptation processes on the work floor reduce the loss of productivity; for example, by prioritizing the more important activities of the patient or the patient's colleagues, or by hiring and training new employees. Although these adaptation processes are complex, the way the FCA is usually applied in practice is simply by limiting the period of production loss to, at most, the friction period (i.e., the time needed to hire a new employee).

The productivity losses estimated by the HCA and the FCA can differ substantially, ranging from potentially decades of loss for the HCA to at most months of loss for the FCA. The HCA overestimates the true productivity loss by disregarding the adaptation processes that reduce the loss to society. In contrast, the FCA underestimates the loss by considering only the loss to the patient's employer. Unless the new employee was previously unemployed, the original vacancy starts a chain of vacancies, friction periods and production losses.

\section{Subgroups in health policy}

Productivity costs can be disregarded in health economic assessments with good reason [2]. A health minister with a finite health budget may aim to maximize total health in her country, regardless of wider consequences. Yet, from a societal perspective, it does make sense to consider healthcare costs less of a problem when they are compensated by savings on productivity. Moreover, 
considering costs in health policy is not a priori unethical, because health is not the only relevant objective and because money is a proxy for how we spend our time and efforts.

Nevertheless, the inclusion of productivity costs in economic assessments does raise some ethical concerns. In particular, it can be at odds with equity of access to healthcare. Equity of access is a central objective of many healthcare systems. By taking productivity into account, health improvement and health care are considered more relevant for patients with a job than for patients without a job. However, considering productivity costs does not necessitate that access to care should be withheld from those without favourable productivity. Economic assessments are usually performed for mixed patient populations, comprising patients with and without jobs. By averaging over the entire population, those without jobs profit as well from the savings on productivity among those with jobs. This could make care accessible for the entire population that would otherwise be considered too expensive.

The paper by Pearce et al. [1] shows that estimated productivity losses differ not only by whether or not the patient has a job, but also by socio-demographic and clinical subgroups. These results suggest that health policy may be targeted at certain groups to maximize cost effectiveness. This suggestion is again at odds with equity of access. Although the subgroups are defined in terms of socio-demographic and clinical variables, these variables in fact serve as proxies for the value of labour. Perhaps it would be most prudent for health economists to refrain from performing subgroup analyses, unless it is clear that differences in cost effectiveness are only due to differences in effectiveness.

Abandoning subgroup analyses within patient populations does not resolve equity concerns across populations, as some patient populations have higher labour participation than others. Taking productivity costs into account is bound to be more favourable to treatments for lumbar disc herniation than to treatments in nursing homes. This disadvantage towards patient populations with low labour participation is ethically comparable to the disadvantage towards patients who are unfortunate to have diseases for which only expensive treatments are available. Both are consequences of taking costs into account, and should prevent health economists from getting too comfortable with their methods.

\section{Research agenda}

According to the paper by Pearce et al. [1], the HCA takes a societal perspective, the FCA takes an employer's perspective, and analyses should either choose the appropriate perspective to match the policy context or pragmatically use both. However, the problem is more fundamental. The reason for including productivity costs in health technology assessment is that health can have societal consequences. Therefore, the appropriate perspective is the societal perspective and neither the HCA nor the FCA properly reflect that societal perspective: full employment and readily available surplus capacity are both unrealistic assumptions. Moreover, both approaches often ignore unpaid labour and efficiency while at work [2, 3].

The FCA was developed in reaction to the deficiencies of the HCA. The FCA has been accused of lacking a theoretical foundation, but instead should be complimented for its effort to incorporate the actual adaptation processes on the work floor; processes that have so far been too complex to be captured in abstract theory. The next phase for the research agenda should be to bridge the gap between both approaches. One step in that direction could be to explore the chain of vacancies, from the initial patient's vacancy to the last vacancy that is filled by a previously unemployed person. Studying that chain is complex:

- tracking the chain requires individual data over long periods of time;

- for each initial patient, it requires the collaboration of a chain of employers and employees that each need to be found and convinced to participate in the study;

- contrary to the initial vacancy, the productivity loss in following vacancies may be reduced by the duration of the term of notice;

- the level of unemployment influences both the duration of the friction period and the probability that a vacancy is filled by a previously unemployed person;

- the friction periods used by Pearce et al. [1] range from 13 weeks for professional occupations to 10 weeks for manual and service occupations. Similarly, the probability of replacement by a previously unemployed person may differ by occupation type.

This type of data may again not allow for abstract theory. Yet, even a rough estimate of the length of the chain would already be useful to suggest a reasonable middle of the bridge between HCA and FCA.

Conflict of interest None.

\section{References}

1. Pearce AM, Hanly P, Timmons A, Walsh PM, O’Neill C, O'Sullivan E, et al. Productivity losses associated with head and neck cancer using the human capital and friction cost approaches. Appl Health Econ Health Policy. 2015. doi:10.1007/s40258-0150155-8. 
2. Knies S, Severens JL, Ament AJ, Evers SM. The transferability of valuing lost productivity across jurisdictions: differences between national pharmacoeconomic guidelines. Value Health. 2010;13(5):519-27.
3. Krol M, Brouwer W. How to estimate productivity costs in economic evaluations. Pharmacoeconomics. 2014;32(4):335-44. 